\title{
0528 TRAFFIC INJURY PREVENTION IN CLUJ-NAPOCA, ROMANIA
}

R M Chereches*, D Rus, N Ajtai, E 0 Sirlincan Correspondence: Babes Bolyai University, Institute for Social Research, Center for Health Policy and Public Health, 71 Traian Mosoiu str, Cluj-Napoca 400132, Romania

\subsection{6/ip.2010.029215.528}

Low and middle income countries still lack proper surveillance such as registries that document traffic fatal and non-fatal injuries. Literature indicates that traffic injuries prevention can be enhanced based on rigorous documentation and reliable data. The Romanian Road Safety Plan is a national centralised program, offering little room for local initiative and evidence based approaches. The traffic injury prevention measures implemented so far involve very few health professional and disregard new technologies. An innovative pilot-project conducted in Cluj-Napoca, Romania used police reports and emergency department data to draw a map of accidents using Global Information Systems (GIS) technology. According to the Police Department Database pedestrian injuries account for $70 \%$ of traffic related injuries. More than a third of the traffic crashes occurred under normal weather and road conditions, during daylight and on dry road. GIS has been used to identify traffic injury hot spots with the primary scope of developing future injury prevention strategies in Cluj-Napoca, Romania. This local need based technologically enhanced initiative, uses GIS to assess high risk areas of the city and to allow the local Police Departments to take corrective actions. The digital map enables to locate precisely the hotspots from the city and to develop a need-based intervention plan. GIS has proved to be a successful tool, specific hotspots being detected and mapped. Further research is needed to determine environmental related causes. Furthermore, population based educational interventions are encouraged. 